\title{
Molecular Characterization of Fusarium Graminearum Virus 2 Isolated from Fusarium graminearum Strain 98-8-60
}

\author{
Jisuk Yu ${ }^{1}$, Kyung-Mi Lee ${ }^{1}$, Moonil Son ${ }^{1}$ and Kook-Hyung Kim ${ }^{1,2 *}$ \\ ${ }^{\prime}$ Department of Agricultural Biotechnology and Center for Fungal Pathogenesis; ${ }^{2}$ Research Institute for Agriculture and Life \\ Sciences, College of Agriculture and Life Sciences, Seoul National University, Seoul 151-921, Korea
}

(Received on March 11, 2011; Revised on May 17, 2011; Accepted on June 10, 2011)

Fusarium graminearum virus 2 (FgV2) infects Fusarium graminearum strain $98-8-60$ and has at least five segments of double-stranded RNAs (dsRNAs), denoted as dsRNA-1 to dsRNA-5. In this study, the genome of FgV2 was sequenced and its phylogenetic relationship with other mycoviruses was analyzed. The lengths of FgV2 dsRNAs 1-5 ranged from 2414 to 3580 base pairs (bp). The $5^{\prime}$ and $3^{\prime}$ untranslated regions (UTRs) are highly conserved, and each dsRNA segment had 78-105 and 84-306 bp of 5' and 3' UTRs, respectively. Each dsRNA segment contained a single open reading frame (ORF). Computer analysis of dsRNA-1 revealed a putative open reading frame (ORF) that shows high sequence identity with an RNA-dependent RNA polymerase (RdRp) containing eight conserved motifs. dsRNAs 2-5 also each contain one putative ORF coding for products of unknown function. The sequences of FgV2 dsRNA-2 and dsRNA-3 have significant sequence identity with Magnaporthe oryzae chrysovirus 1 (MoCV1) dsRNA-3 and -4 , respectively. When compared to other dsRNA mycoviruses in a phylogenetic analysis of the putative RdRp protein, FgV2 was found to form a distinct virus clade with Aspergillus mycovirus 1816 and MoCV1 in the family Chrysoviridae.

Keywords : Chrysoviridae, Fusarium graminearum virus 2, Mycovirus

The Fusarium graminearum clade [Teleomorphs: Gibberella species] is an important plant pathogens that cause head and seedling blight of small grains such as wheat and barley, stalk and ear rot of corn, and stem rot of carnation and thus cause enormous economic losses and mycotoxin contamination in cereals (Cook, 1981; Lee et al., 2010). In previous research, we determined the diversity and incidence of mycoviruses that infect $F$. graminearum clade based on the presence of dsRNAs (Chu et al., 2004).

\footnotetext{
*Corresponding author.

Phone) +82-2-880-4677, FAX) +82-2-873-2317

E-mail)kookkim@snu.ac.kr
}

Among the viruses that infect the $F$. graminearum clade, Fusarium graminearum virus 1-DK21 (FgV1-DK21) has been reported to be associated with reduced mycelial growth, increased pigmentation, and reduced virulence on wheat plants (Chu et al., 2004). In contrast, the following three dsRNA viruses, which were isolated from F. graminearum strains 98-8-60 and DK3, infect their host asymptomatically: Fusarium graminearum virus 2 (FgV2), Fusarium graminearum virus 3 (FgV3), and Fusarium graminearum virus 4 (FgV4) (Chu et al., 2004; Yu et al., 2009). Complete genome sequence analysis and molecular characterization of FgV1-DK21 indicated that its putative genomic organization and gene expression are similar to those of the plant potex-like ssRNA virus group, although the sequence similarity was low for both the RNA-dependent RNA polymerase (RdRp) domain and the full-length ORF1 (Kwon et al., 2007). In contrast, analysis of both genome organization and phylogeny indicated that $\mathrm{FgV3}$ was closely related to members of the families Totiviridae and Chrysoviridae whereas FgV4 formed a distinct clade with the family Partitiviridae (Yu et al., 2009).

A similar analysis has not been performed for $\mathrm{FgV} 2$ because the complete genome sequence of FgV2 has not been reported. Here, we describe the nucleotide sequence of FgV2 infecting F graminearum strain 98-8-60. We report that $\mathrm{FgV} 2$ has at least five dsRNA segments. Sequence analysis revealed that each dsRNA segment encodes a single open reading frame (ORF) and contains highly conserved sequences at both $5^{\prime}$ and $3^{\prime}$ ends.

F. graminearum strain 98-8-60 containing dsRNAs was obtained from barley (Chu et al., 2004). Viral dsRNAs were extracted from mycelia using CF-11 cellulose chromatography (Chu et al., 2004). The number and presence of dsRNAs were determined by $1 \%$ agarose and $6 \%$ polyacrylamide gels. cDNA synthesis and amplification using random primers were conducted as previously described (Kwon et al., 2007; Urayama et al., 2010). The amplified polymerase chain reaction (PCR) product was visualized on $1 \%$ agarose gel by ethidium bromide (EtBr) staining. PCR products were purified using a QIAquick PCR purification 
kit (Qiagen, Hilden, Germany) and subsequently cloned into the pGEM-T Easy Vector (Promega, Madison, WI, USA). Based on the dsRNA sequences obtained, dsRNAspecific primers were designed to amplify overlapping fragments and were used for RT-PCR. The 5' and 3' untranslated regions (UTRs) of each dsRNA segment were identified by the RACE method (Cold Spring Harbor Laboratory Press, 2005). All clones were sequenced at least three times in both orientations using universal and specific PCR primers at the National Instrumentation Center for Environmental Management, Seoul National University, Korea. Each dsRNA segment was identified by Northern hybridization using the cloned cDNAs as probes. Nucleotide sequence data were assembled using the Seqman program in DNASTAR. A sequence similarity search of viral RNAs was conducted with the NCBI BLAST program. The complete nucleotide sequences of dsRNA segments 1 to 5 of FgV2 were deposited in GenBank (accession numbers HQ343295 to HQ343299, respectively). The alignment of putative RdRp amino acid sequences of FgV2 dsRNA-1 and selected dsRNA mycoviruses was performed using BioEdit sequence alignment editor (Version 7.0.9) (http:// www.mbio.ncsu.edu/bioedit/bioedit.html) and GeneDoc programs (http://www.nrbsc.org/gfx/genedoc/). Phylogenetic analysis of RdRp regions of dsRNA mycoviruses including FgV2 were carried out by the MEGA program, version 4.0 (Kumar et al., 2008).

Previously, we reported that dsRNA mycovirus infecting $F$. graminearum strain 98-8-60 contained two similar sized dsRNAs of ca. 3.5 and $2.5 \mathrm{kbp}$ with occasional occurrence of one additional smaller dsRNA of approximately $2.3 \mathrm{kbp}$ based on separation on a 1\% agarose gel (Chu et al., 2004).
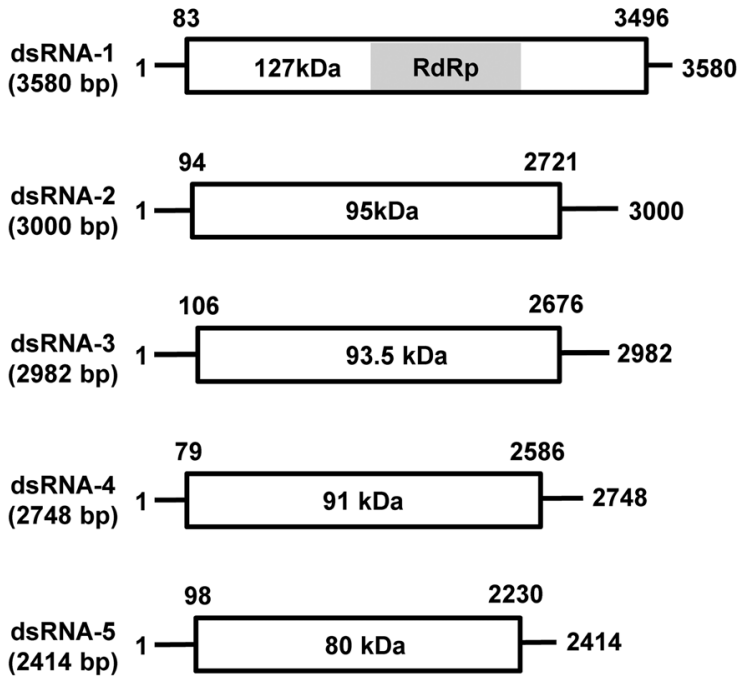

Fig. 1. Genome organization of FgV2. The rectangular box represents the ORF defined by sequence analysis, and the molecular weights of the proteins encoded by each ORF are indicated in the boxes. The grey bar in the center of the dsRNA-1 ORF represents the conserved viral RNA-dependent RNA polymerase (RdRp) domain. The un-translated regions are indicated by a single line. The nucleotide positions of the initiation and termination codons are indicated above the border of the ORF.

In the current study, however, at least five dsRNA segments were always observed on a $6 \%$ polyacrylamide gel, and one or two additional smaller dsRNAs were infrequently observed (data not shown). Because these smaller dsRNAs are unstable in both repeated subculturing and single spore isolation, it is possible that these dsRNAs may be satellite RNAs, and thus they were not regarded as genome segments of FgV2. Accumulation of smaller satellite dsRNA(s)

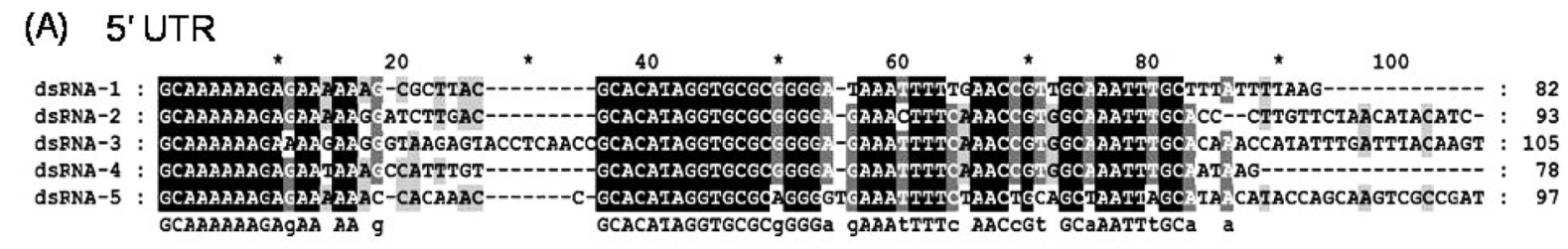

\section{(B) 3'UTR}

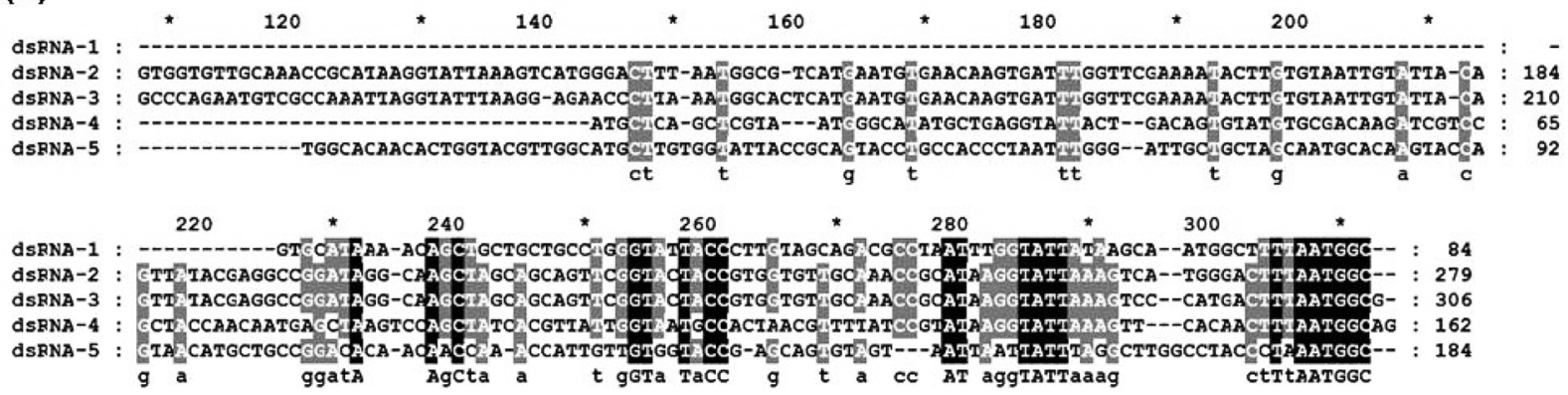

Fig. 2. Conserved sequence elements in FgV2 dsRNA segments. Sequences of the $5^{\prime}$ UTR (A) and 3' UTR (B) of each dsRNA segment that are identical are reverse highlighted. Black, dark grey, and light grey shading levels represent $100 \%, 80 \%$, and $60 \%$ nucleotides conservation, respectively. 
has also been reported in other dsRNA mycoviruses including Saccharomyces cerevisiae virus $L-A$ and Ustilago maydis virus-H1 (Pearson et al., 2009).

In this study, we designated the five dsRNAs that were consistently detected in infected $F$. graminearum strain 988-60 as dsRNA-1 to dsRNA-5. The sizes of dsRNAs $1-5$ ranged from 3580 to $2414 \mathrm{bp}$ (Fig. 1). The 5' and 3' UTR of these dsRNA segments were 78-105 and 84-306 bp, respectively. Direct comparison of the 5' UTR sequences of dsRNA segments 1 to 5 revealed a stretch of conserved sequences including GCAAAAAAGA at the 5' terminus of each dsRNA segment (Fig. 2A). The 3' UTR of the five genomic dsRNAs also contained conserved sequences (Fig. 2B). The ${ }^{32} \mathrm{P}-\mathrm{dCTP}$ labeled cDNA clones containing DNA sequences corresponding to each dsRNA segment were used as probes for Northern blot analysis to determine sequence similarity and to detect the presence of virusspecific RNAs in infected cells. Each probe specifically hybridized to each dsRNA in FgV2 infected $F$. graminearum

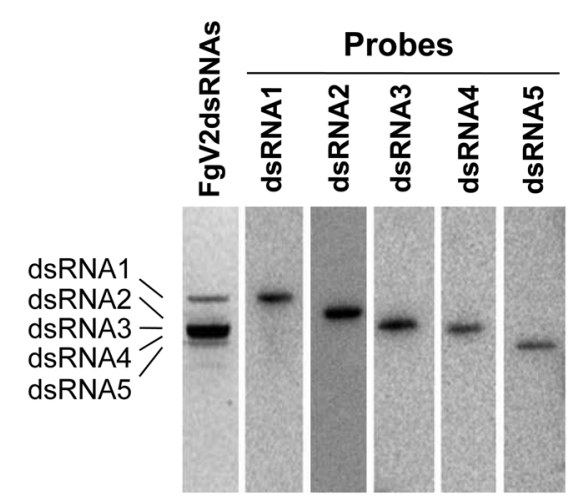

Fig. 3. Northern blot analysis. Purified dsRNAs were separated on a $1 \%$ agarose gel, stained with ethidium bromide, and blotted to Hybond-N+ membrane. ${ }^{32} \mathrm{P}-\mathrm{dCTP}$ labeled DNA probes specific for each dsRNA region (nt 1257-1674, 747-1284, 23392766, 1152-1512, and 203-682 of dsRNAs 1 to 5, respectively) were prepared and used for hybridization. The membrane was reused for the blot to detect different segments of FgV2 by using multiple probes. Each of the dsRNA specific probes was stripped completely before rehybridizing with another probe.

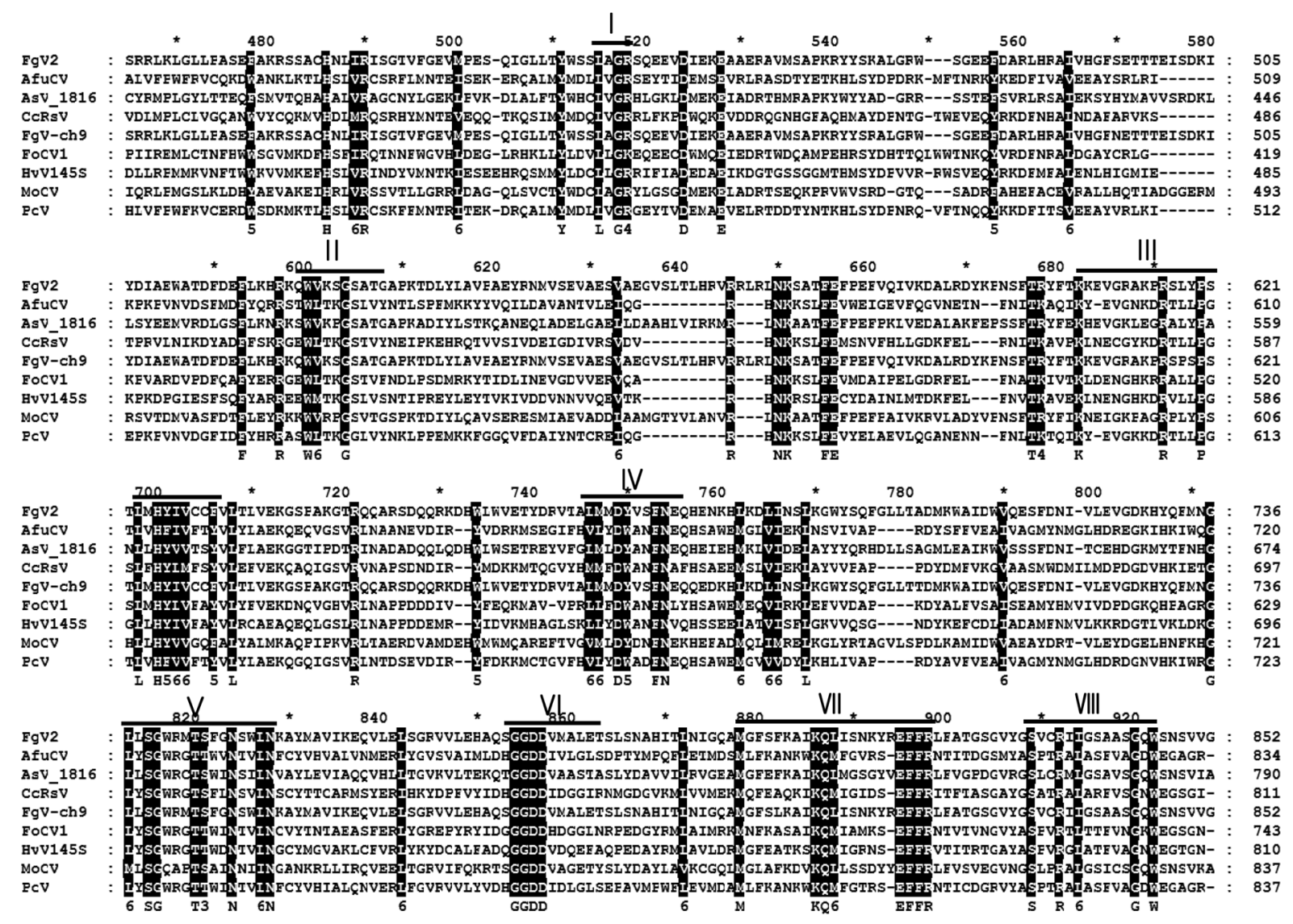

Fig. 4. Amino acid sequence alignment including eight conserved RdRp motifs of FgV2 with those of other dsRNA mycoviruses in the Chrysoviridae family. Numbers I-VIII indicate the eight conserved motifs in RdRps. The positions of the amino acid sequences are indicated on the right. Abbreviations of virus name and Genbank accession numbers are the following: AfuCV, Aspergillus fumigatus chrysovirus (FN178512); AsV1816, Aspergillus mycovirus 1816 (EU289896); CCRSV, Cherry chlorotic rusty spot chrysovirus (AJ781397); FgV2, Fusarium graminearum virus 2 (HQ343295); FgV-ch9, Fusarium graminearum mycovirus-China 9 (HQ228213); FoCV1, Fusarium oxysporum chrysovirus 1 (EF152346); HvV145S, Helminthosporium victoriae virus 145S (AF297176); MoCV1, Magnaporthe oryzae chrysovirus 1 (NC014462); PcV, Penicillium chrysogenum virus (AF296439). 
(Fig. 3). This result indicated that each segment has unique sequences.

Computer analysis of FgV2 dsRNAs 1-5 showed that each dsRNA contained a single ORF encoding 127-, 95-, 93.5-, 91-, and 80-kDa proteins, respectively (Fig. 1). Recently, the complete genome sequence of FgV-ch9 which is isolated from cereals in China has been reported (Darissa et al., 2011). Interestingly, sequence analysis revealed that FgV2 shares overall nucleotide sequence identity of 91\%, $97 \%, 96 \%, 88 \%$ and $85 \%$ with FgV-ch9 for dsRNA-1 to -5 ,

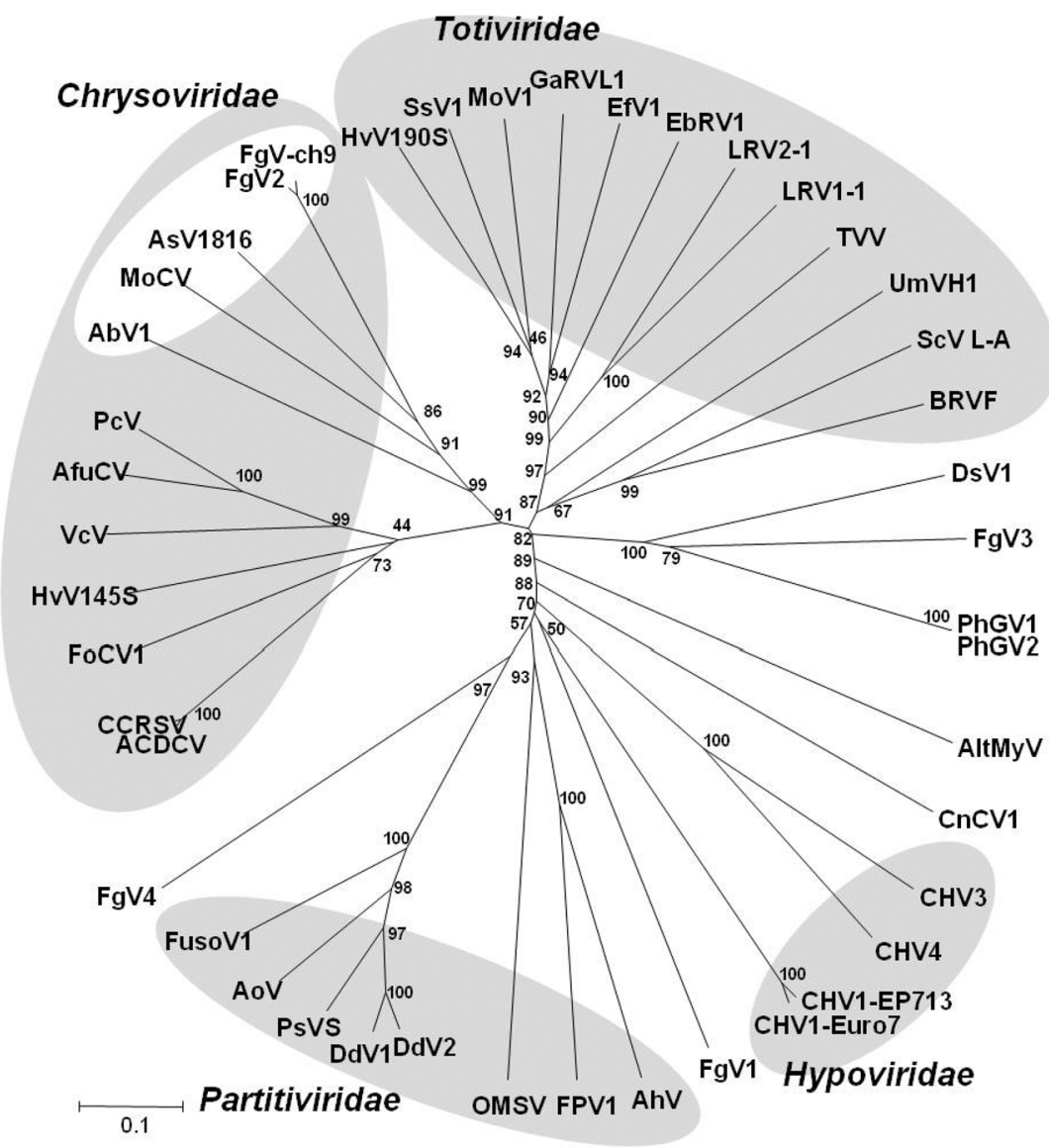

Fig. 5. A phylogenetic tree of FgV2 and members of selected mycovirus families based on amino acid sequences of RdRp. Phylogenetic trees were constructed by MEGA version 4.0 program using the neighbor-joining method with 1000 bootstrap replicates. The numbers at each branch display bootstrap values (1000 replicates). Closely related mycoviruses are circled. Abbreviations of virus name and Genbank accession numbers are the following: ABV1, Agaricus bisporus virus 1(X94361); ACDCV, Amasya cherry disease associated chrysovirus (AJ781166); AfuCV, Aspergillus fumigatus chrysovirus (FN178512); AhV, Atkinsonella hypoxylon virus (NC003470); AltMyV, Alternaria alternata dsRNA mycovirus (AB368492); AoV, Aspergillus ochraceous virus (DQ270031); AsV1816, Aspergillus mycovirus 1816 (EU289896); BRVF, Black raspberry virus F (EU082131); CCRSV, Cherry chlorotic rusty spot chrysovirus (AJ781397); CHV1-EP713, Cryphonectria hypovirus1 EP713 strain (NC001492); CHV1-Euro7, Cryphonectria hypovirus1 Euro strain (AF082191); CHV3, Cryphonectria hypovirus 3 (AF188515); CHV4, Cryphonectria hypovirus 4 (AY307099); CnCV1 (GQ290650); Cryphonectria nitschkei chrysovirus 1 bs131 strain; DdV1, Discula destructiva virus 1 (NC002797); DdV2, Discula destructiva virus 2 (NC003710); DsV1, Diplodia scrobiculata virus 1 (EF568774); EbRV1, Eimeria brunetti RNA virus 1 (AF356189); EfV1, Epichloe festucae virus 1(AM261427); FgV1, Fusarium graminearum virus 1-DK21 (AY533037); FgV2, Fusarium graminearum virus 2 (HQ343295); FgV-ch9, Fusarium graminearum mycovirus-China 9 (HQ228213); FgV3, Fusarium graminearum virus 3 (GQ140626); FgV4, Fusarium graminearum virus 4 (GQ140627); FoCV1, Fusarium oxysporum chrysovirus 1 (EF152346); FpV1, Fusarium poae virus 1 (AF047013); FusoV1, Fusarium solani virus 1 (D55668); GaRVL1, Gremmeniella abietina RNA virus L1 (AF337175); HvV145S, Helminthosporium victoriae virus 145S (AF297176); HvV190S, Helminthosporium victoriae virus 190S (U41345); LRV1-1, Leishmania RNA virus 1-1 (M92355); LRV2-1, Leishmania RNA virus 2-1 (U32108); MoCV1, Magnaporthe oryzae chrysovirus 1 (NC014462); MoV1, Magnaporthe oryzae virus 1 (AB176964); OMSV, Oyster mushroom spherical virus (AY182001); PcV, Penicillium chrysogenum virus (AF296439); PhGV1, Phlebiopsis gigantea mycovirus1 (AM111096); PhGV2, Phlebiopsis gigantea mycovirus 2 (AM111097); PsVS, Penicillium stoloniferum virus S (AM040138); ScVL-A, Saccharomyces cerevisiae virus L-A (U01060); SsV1, Sphaeropsis sapinea RNA virus 1 (AF038665); TVV, Trichomonas vaginalis virus (U08999); UmVH1, Ustilago maydis virus H1 (U01059); VcV, Verticillium chrysogenum virus (HM00467). 
respectively. Putative amino acid sequences of the entire ORF from the dsRNA-1 to dsRNA-5 showed $98 \%, 99 \%$, $99 \%, 94 \%$ and $83 \%$ of identity with those from the FgVch9, respectively. This result suggests that FgV2 and FgVch9 are closely related each other. Comparative analysis with sequences in the databases revealed sequence similarity to RdRp of other mycoviruses (Bruenn, 1993; Fig. 4) including Aspergillus mycovirus 1816 (AsV1816; Hammond et al., 2008) and Magnaporthe oryzae chrysovirus 1 (MoCV1; Urayama et al., 2010). FgV2 dsRNA-2 does not contain a specific domain suggesting a function but the deduced amino acid sequence of FgV2 dsRNA-2 showed relatively high sequence identity (29\%) with MoCV1 dsRNA segment 3 . In addition, the deduced amino acid sequences of dsRNA-3 of $\mathrm{FgV} 2$ showed $23 \%$ and $22 \%$ sequence identity with MoCV1 dsRNA segment 4 and La France disease virus L3 segment, respectively. It was reported that members and tentative members of Chrysoviridae include consensus amino acid sequences of the C-terminal region that contain the putative motifs of the core of the ovarian tumour gene-like superfamily of predicted protease (Jamal et al., 2010). Sequence alignment of FgV2 dsRNA-3 with members of Chrysoviridae indicated that dsRNA-3 also contained motifs III and IV (data not shown). Interestingly, alignment of the amino acid sequences of FgV2 dsRNA-3 showed about $23 \%$ identity to the $\mathrm{L} 3$ capsid protein (CP) of Agaricus bisporus virus 1 (ABV1). This result indicates that FgV2 dsRNA-3 most likely codes for the CP. In this regard, it is worth noting recent observation that dsRNA3 of FgV-ch9 might code for the CP (Darissa et al., 2011). Sequence analysis of dsRNA-4 and dsRNA-5 shows that they consist of 2748 and $2414 \mathrm{bp}$, respectively, and each contain a single ORF encoding a product of unknown function. Nucleotide and the deduced amino acid sequences of dsRNA-4 did not show any significant homology with other known sequences. The deduced amino acid sequence of dsRNA-5 contains a zinc finger protein domain at Cterminal region with homology only to the equivalent dsRNA-5 product of FgV-ch9 (Darissa et al., 2011).

To elucidate the phylogenetic relationships of FgV2 with other reported mycoviruses, a phylogenetic analysis, based on the amino acid sequence of the RdRp domain, was conducted using FgV2 dsRNA-1 and other selected dsRNA mycoviruses belonging to the families Totiviridae, Chrysoviridae, Partitiviridae, and Hypoviridae including FgV-ch9 dsRNA-1. On the basis of RdRp protein sequences, FgV2 forms a separate clade with FgV-ch9, MoCV1, AsV1816, and ABV1 in the Chrysoviridae family (Fig. 5). Although all reported chrysoviruses have four dsRNA segments (ICTV, 2010) and form nonenveloped isometric particles, the FgV2 genome is composed of at least five dsRNA segments. FgV2, however, contained a potential CP gene in
dsRNA-3 segment. This is in agreement with the recent finding that FgV-ch9, another potential member of the Chrysoviridae family, formed isometric virus-like particles of 35-40 nm in diameter (Darissa et al., 2011). Altogether, sequence comparison and phylogenetic analysis of $\mathrm{FgV} 2$ suggests that $\mathrm{FgV} 2$ and $\mathrm{FgV}$-ch9 are closely related isolates of the same species and these two virus isolates belong to a new and as yet unassigned genus of mycoviruses that is closely related to the chrysoviruses.

\section{Acknowledgements}

This research was supported by a grant from the Center for Fungal Pathogenesis (No. 20110000959) funded by the Ministry of Education, Science, and Technology (MEST). JY, KML, and MS were supported by graduate research fellowships from the MEST through Brain Korea 21 Project.

\section{References}

Bruenn, J. A. 1993. A closely related group of RNA-dependent RNA polymerases from double-stranded RNA viruses. Nucleic Acid Res. 21:567-569.

Chu, Y.-M., Jeon, J.-J., Yea, S.-J., Kim, Y.-H., Yun, S.-H., Lee, Y.W. and Kim, K.-H. 2004. Double-stranded RNA mycovirus from Fusarium graminearum. Appl. Environ. Microbiol. 68:2529-2534.

Chu, Y.-M., Lim, W.-S., Yea, S.-J., Cho, J.-D., Lee, Y.-W. and Kim, K.-H. 2004. Complexity of dsRNA mycovirus isolated from Fusarium graminearum. Virus Genes 28:135-143.

Cold Spring Harbor Laboratory Press. 2005. Rapid amplification of 5 ' complementary DNA ends (5' RACE). Nature Methods 2:629-630.

Cook, R. J. 1981. Fusarium diseases of wheat and other small grains in North America. In: Fusarium diseases, biology, and taxonomy, ed. by P. E. Nelson, T. A. Toussoun and R. J. Cook, pp. 39-52. The Pennsylvania State University Press, University Park, United States.

Darissa, O., Willingmann, P., Schafer, W. and Adam, G. 2011. A novel double-stranded RNA mycovirus from Fusarium graminearum: nucleic acid sequence and genomic structure. Arch. Virol. 156:647-658.

Hammond, T. M., Andrewski, M. D., Roossinck, M. J. and Keller, N. P. 2008. Aspergillus mycoviruses are targets and suppressors of RNA silencing. Eukaryot. Cell 7:350-357.

International Committee on Taxonomy of Viruses (ICTV). 2010. Virus Taxonomy: 2009 Release. http://www.ictvonline.org/ virusTaxonomy.asp?version=2009.

Jamal, A., Bignellc, E. M. and Couttsa, R. H. 2010. Complete nucleotide sequences of four dsRNAs associated with a new chrysovirus infecting Aspergillus fumigates. Virus Res. 153: 64-70.

Kumar, S., Nei, M., Dudley, J. and Tamura, K. 2008. MEGA: A biologist-centric software for evolutionary analysis of DNA and protein sequences. Brief Bioinform. 9:299-306. 
Kwon, S.-J., Lim, W.-S., Park, S.-H., Park, M.-R. and Kim, K.-H. 2007. Molecular characterization of a dsRNA mycovirus, Fusarium graminearum virus-DK21, which is phylogenetically related to hypoviruses but has a genome organization and gene expression strategy resembling those of plant potex-like viruses. Mol. Cells 23:304-315.

Lee, S.-H., Lee, J., Nam, Y. J., Lee, S., Ryu, J.-G. and Lee, T. 2010. Population structure of Fusarium graminearum from maize and rice in 2009 in Korea. Plant Pathol. J. 26:321-327.

Pearson, M. N., Beever, R. E., Boine, B. and Arthur, K. 2009. Mycoviruses of filamentous fungi and their relevance to plant pathology. Mol. Plant. Pathol. 10:115-128.

Urayama, S., Kato, S., Suzuki, Y., Aoki, N., Le, M. T., Arie, T., Teraoka, T., Fukuhara, T. and Hiromitsu, M. 2010. Mycoviruses related to chrysovirus affect vegetative growth in the rice blast fungus Magnaporthe oryzae. J. Gen. Virol. 91:30853094.

Yu, J., Kwon, S.-J., Lee, K.-M., Son, M. and Kim, K.-H. 2009. Complete nucleotide sequence of double-stranded RNA viruses from Fusarium graminearum strain DK3. Arch. Virol. 154:1855-1858. 